\title{
A protégé semantic modelling approach for combination correlation of manufacturing service
}

\author{
Feng Xiang*, Jiangchun Liu, \\ Guozhang Jiang and Xiaowu Chen
}

School of Machinery and Automation,

Wuhan University of Science and Technology,

Wuhan 430081, China

Email: 38452712@qq.com

Email: 1248401480@qq.com

Email: 2514045612@qq.com

Email: 48861001@qq.com

*Corresponding author

\begin{abstract}
Cloud manufacturing service model aimed at gathering global resources, optimise the allocation, provide new impetus for the core of manufacturing industry informatisation and the reform, and the service combination technology is a key technology to optimal allocate various resources in manufacturing cloud. However, because the multiple user demands the high system scalability and task complexity, the potential constraint relationship between the various combinations must be taken into account. Focus on the combinable relations researches on combinable relation classification by workflow and strength degree, such as sequential relationships branch/selection relationships, concurrent relationships, multiple selection, synchronous convergence, discriminator, arbitrary cycle, and delay selection. Finally, we use OWL language to build a model of relations ontology for magnetic bearing.
\end{abstract}

Keywords: cloud manufacturing; combination correlation; ontology.

Reference to this paper should be made as follows: Xiang, F., Liu, J., Jiang, G. and Chen, X. (2017) 'A protégé semantic modelling approach for combination correlation of manufacturing service', Int. J. Service and Computing Oriented Manufacturing, Vol. 3, No. 1, pp.13-23.

Biographical notes: Feng Xiang holds a $\mathrm{PhD}$ from the School of Machinery and Automation, Wuhan University of Science and Technology, Wuhan 430081, China. His research areas include service-oriented manufacturing, manufacturing information technology.

Jiangchun Liu holds a BS from the School of Machinery and Automation, Wuhan University of Science and Technology, Wuhan 430081, China. His research areas include service-oriented manufacturing, manufacturing information technology.

Guozhang Jiang holds a PhD from the School of Machinery and Automation, Wuhan University of Science and Technology, Wuhan 430081, China. His research areas include knowledge management and scheduling of iron and steel. 
Xiaowu Chen holds an MS from the School of Machinery and Automation, Wuhan University of Science and Technology, Wuhan 430081, China. His research areas include knowledge management and scheduling of iron and steel.

\section{Introduction}

Cloud manufacturing is committed to solve the manufacturing resources of the global convergence and sharing, realise the optimisation of resource allocation and new service model of the manufacturing new platform (Tao et al., 2011; Tao, 2012; Tong, 2008). It combines the existing advanced manufacturing mode, and the latest research results of the information technology, especially the cloud computing technology, highlight and expand the ideology that 'services as everything', which adhered by cloud computing. It will encapsulate the manufacturing resources which involved the whole life cycle into the cloud manufacturing service, and according to certain rules of the manufacturing cloud services to form a virtual manufacturing environment, and according to the resource requirements to lease. Thus, the key to optimise the allocation of resources lies in the promotion of virtual manufacturing environment construction process and improvement of the overall cloud manufacturing service combination effect. The key technology of cloud manufacturing service composition technology environment is to implement the optimal allocation of manufacturing cloud service oriented (Xuejiao and Xianhui, 2012; Feng et al., 2014 Bohu et al., 2010).

Service composition technology is the core technology of service oriented computing, is also widely used in service-oriented manufacturing ( $\mathrm{SOM}$ ) system. At present, the most widely used and researched is web service composition, which concretely divides as the three stages of plan, definition and implementation. Through the dynamic semantic analysis and the automatic matching service, and reduce the unnecessary artificial intervention, to make the manufacturing service automation more easily to implement. In the process of variety services combination, we need to fully consider the combination relationship between the services, in the face of increasingly complexity and volatility of the services demand, each kind of services are not in sequence to carry on but need the complexity combination to completed the established tasks, therefore, it is necessary to study the combinable relationship and transformation of cloud manufacturing services, and better serve as the cloud manufacturing services composition.

In recent ten years, service combination and optimisation problem caused the extensive concern of the domestic and foreign scholars. Through the part of the service composition and its literature research and analysis, the existing research about the service composition and its combination can be roughly divided as three:

1 Description of framework for service composition and its combination research.

Song et al. (2011) and Chunyang et al. (2015) aim at the heterogeneous problems in the combination process of the Web services which provided by different web service providers, they proposed service composition implementation framework based on semantic web. The framework in detail elaborated the semantic web service selection strategy, combination relation setting, and the key technology in composition implementation process such as data transmission and invocation 
mechanism and so on which among the members of the web services composition internal.

2 The research of service composition mechanism, ideology and algorithm. Changjun (2006) proposed a new service composition relationship description method, it can be combined based on the graph description method and based on the Petri net, the advantages of semantic description method are the users can conveniently describe the combination relationships. Liu (2012) aims at the context of multi task and complexity multi task request mode in cloud manufacturing, with the service combination and optimisation problem as the research object, with the improvement of the overall effect of resources optimisation allocation as the research goals, to discuss the key technology of service combination and optimisation based on multi task in cloud manufacturing environment. Tao et al. (2008) studies the composition relationship of the service network based on the combination service network that is the scale-free network.

3 Description of services composition language research. Nan (2010) proposed a semantic web service composition method based on diagram, the web service composition process use the semantic technology, to solve the low correct rate problem of the traditional service combination method. To put forward a service matching method based on the parameters of the semantic similarity, study how to combine the domain ontology technology and semantic web service description technology, and through calculate the concept similarity to solve the problem of service matching, in order to improve the rationality of the service selection (Liu, 2012; Qing, 2008; Zhiyao et al., 2006; Zhiwei et al., 2010; Zhen et al., 2013).

At present, the cloud manufacturing service combination and optimisation research has a lot of, but there is little to systematic describe the combinable correlation of the manufacturing service. Therefore, this paper is based on the background of cloud manufacturing, in order to understand the combinable relationship and its classification (Changxia et al., 2009; Ardagna and Pernici, 2007; Tao et al., 2008; Zhang et al., 2010; Guo et al., 2010). Based on OWL-S semantic, use protégé software to establish the cloud manufacturing service combinable relationship model. Aimed at variety of tasks request mode in cloud manufacturing environment, according to certain rules to combine the single function of cloud manufacturing service, and formed cloud manufacturing combination relations with internal logic process, and better provide personalised services for the cloud manufacturing terminal users.

\section{Combinable relation description}

\subsection{The ontology definition of the combinable relation}

The combinable relation means a relationship between a unit with the other units in the same sequence, or co-occurrence of all components of the relationship, or the words in combinable relation must to meet some of syntactic and semantic conditions. In manufacturing services, when the number of service to building the combinable applications, such as a bearing assembly requires taking into account all the parts related the combination services. The combination can be used to as the resource ontology 
concepts, an ontology was not originally used in modelling field, it just began to appear in the field of philosophy, is defined as: 'a systematic description of the objective existence in the world', is an system explanation or illustration of the objective existence in the world, which is concerned with the abstract nature of the objective reality. Later, a number of different definitions are given by more and more people who study the ontology in the field of information systems, knowledge systems (Tao et al., 2012; He, 2008; Sheng et al., 2008; Kai et al., 2014; Feng, 2013; Xudong, 2013; Yiming, 2013). And now it is generally believed that ontology concept should include four aspects:

a The conceptual model (conceptualisation) refers to the model obtained by the relevant concepts of some phenomena in the objective world.

b The explicit refers to the concept in use and its constraints have a clear definition.

c The formalisation (formal) refers to the ontology, which is readable by computer (could be processed by the computer).

d The sharing refers to the user common recognition of the knowledge in ontology, reflecting the recognised concept set in the relevant field.

\subsection{Combinable relation classification by workflow}

In the model of work-flow, the logical connection between the services are constrained by the time sequence of semantic services, which determines the call order of services, the most basic work-flow divided as sequential, concurrent and select.

a Sequential relationships. If the service A and service B performance as the sequential relationship, then, only when after the A executed, $B$ can be executed, that is, the service A to serve as a precursor of B service.

b Branch/selection relationships. If the service $\mathrm{B}$ and service $\mathrm{C}$ belong to a branch relationship, only when the service $\mathrm{B}$ execution conditions are met, the service $\mathrm{B}$ can be executed, if the service $\mathrm{C}$ execution conditions are met, then the services $\mathrm{C}$ can be executed.

c Concurrent relationships. If the service $\mathrm{B}$ and service $\mathrm{C}$ belong to a concurrent relations, then the service $\mathrm{C}$ and service $\mathrm{B}$ can be executed simultaneously, and will not interfere with each other.

These are the most basic logical relationships in the workflow model, through combine the basic relationships can generate more complicated and changeable logical relationships.

d Multiple selection. The combinable relationship can be selected one or more services based on the decision conditions to execute

e Synchronous convergence. In the process of service composition, a control flow is synthesised by many services, if a number of services are executed, then those services are need to be executed simultaneously

f Discriminator. In the service composition relations, multi services are started simultaneously, as long as one of the service is executed, then you can start the next service, and the rest executing services will be ignored 
g Arbitrary cycle. This combinable relationship refers to the services can be recycled

h Delay selection. This combinable relationship refers to that based on the environmental conditions or resource selections to start one of the services.

Figure 1 Sequential relations combinable relation classification by workflow
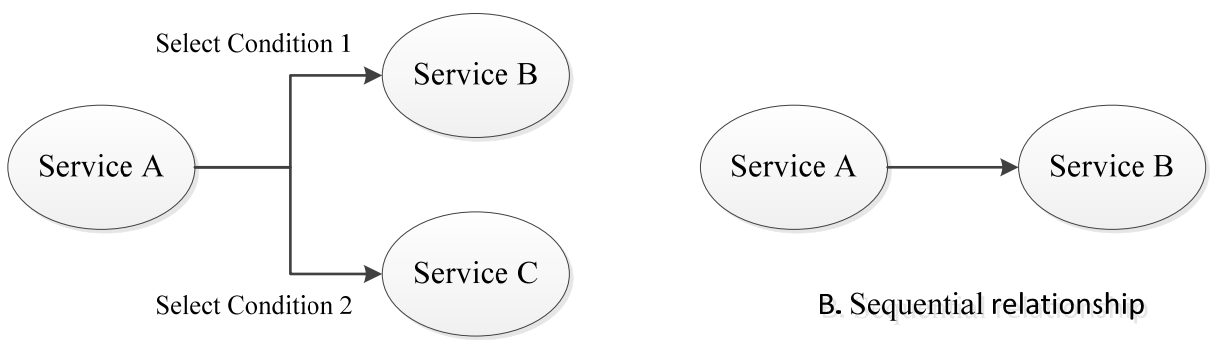

B. Sequential relationship

A. Selection relationship
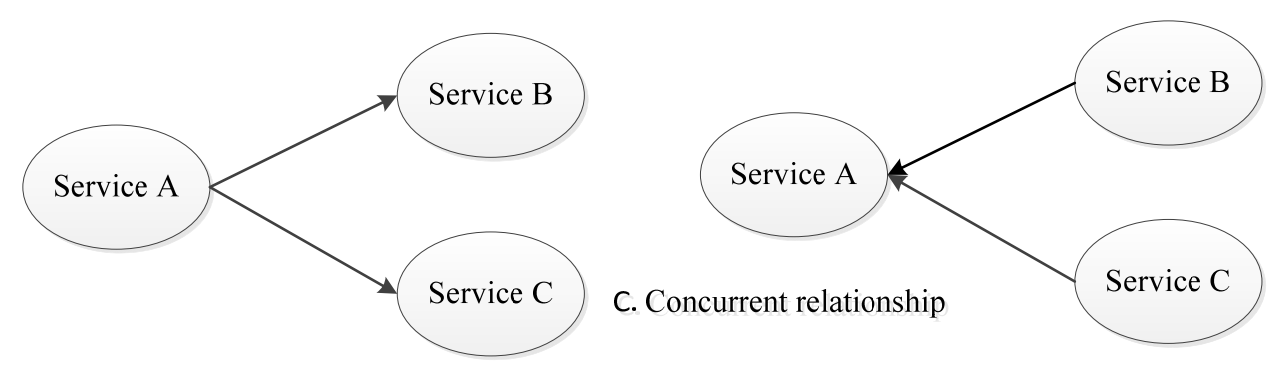

\subsection{Combinable relation classification by strength degree}

In the service composition process, sometimes there will be such a situation, a service fails to meet the manufacturing service demand, then whether the service can be replaced by the other services to ensure the service composition quality, which means the important degree of a service to the service composition, we can use combinable relation degree to represent the importance of the service.

Table 1 Neighbour service composition relationship

\begin{tabular}{lcc}
\hline Combinable relations & Symbol & Combination relation degree \\
\hline Equivalent or competition combination & $S p_{i} \Leftrightarrow S p_{j}$ & $\mathrm{~F} 1$ \\
Equivalent input combination & $S p_{i} \succ S p_{j}$ & $\mathrm{~F} 2$ \\
Equivalent output combination & $S p_{i} \prec S p_{j}$ & $\mathrm{~F} 3$ \\
Partial pre-order combination & $S p_{i} \supset S p_{j}$ & $\mathrm{~F} 4$ \\
Partial successor combination & $S p_{i} \subset S p_{j}$ & $\mathrm{~F} 5$ \\
\hline
\end{tabular}

In generally, combinable relation degree can be divided into strong combinable relation and weak combinable relation, strong combinable relations refers to the service in each 
part of the service composition cannot be replaced, and cannot be lack anyone. The weak combinable relations refers to a service can be replaced by other services. According to the actual need, they can also be further subdivision for the various grades. The following Table 1 defines the strength of the neighbour service composition:

The definition of combinable relation degree in Table 1 can be valued according to the actual manufacturing service composition. In this way, it makes the service composition process automation degree higher, because the specific numerical values are more convenient for computer to implement.

\subsection{Transformation of the combinable relations}

In the process of manufacture service composition, the relationship between a few services have been defined may change due to the actual situation, or to combine a several services composition itself to construct another combinable relations or more complex combinable relations, so as to form the service transformation of the combinable relations, which is shown in Figure 2.

Figure 2 Transformation of service composition

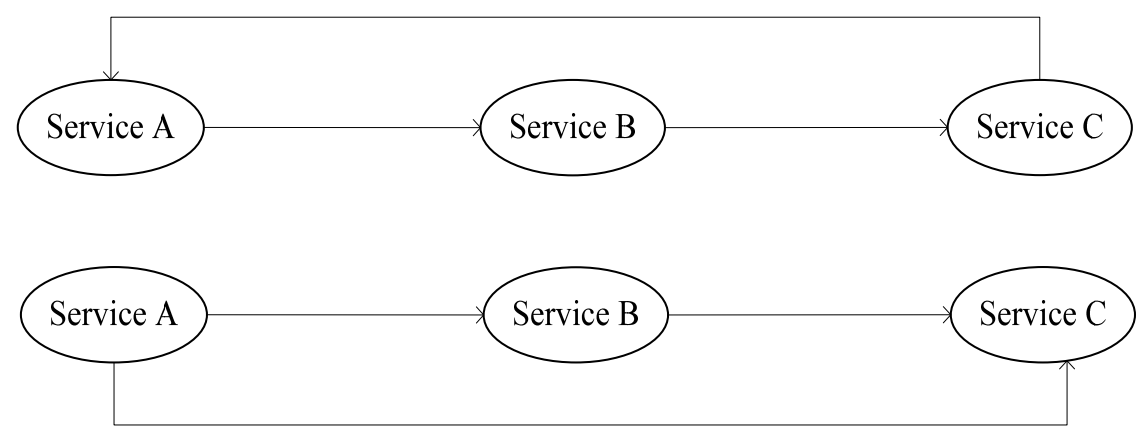

In the process of service composition, service $\mathrm{A}$ and $\mathrm{B}$ are sequential relationship, service $\mathrm{B}$ and $\mathrm{C}$ are sequential relationship too, but if the service $\mathrm{C}$ is followed by service $\mathrm{A}$, the services $\mathrm{A}, \mathrm{B}$, and $\mathrm{C}$ become the relationship of cyclic; if service $\mathrm{B}$ is followed by service $\mathrm{A}$ and immediately service $\mathrm{C}$ is followed by service $\mathrm{A}$, so service $\mathrm{B}, \mathrm{C}$ are also constitute the concurrent relations.

\subsection{Succession of the combinable relations}

In language, succession usually refers to the function of a class (called sub classes, sub interface) succeed another class (called parent classes, parent interface), and it can be improved the ability of its new functions, succession is the most common relationship between class and class or interface and interface, as it shown in Figure 3.

In the service composition process, the service $\mathrm{B}$ and service $\mathrm{C}$ to a sub class concurrent relations of service $A$ will success the service $\mathrm{D}$ and service $\mathrm{E}$ to a parent class concurrent relations of service B. In fact, the general success relations are the same, a small part of the succession have original innovation based on the parent class. 
Figure 3 The succession of the combinable relations

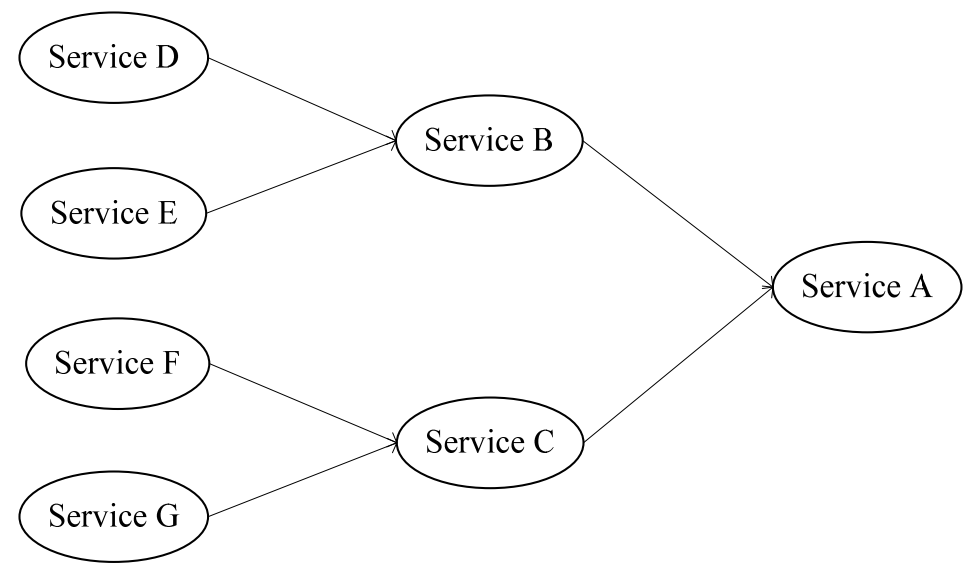

\subsubsection{Inference of the combinable relations}

Combinable relation of reasoning, just as its name implies, in the bases of the cloud manufacturing service system, on speculation of the front relationship between service composition and given data based on the background of the service composition relations, which is not only related to the comprehensive application relationship of the intelligent service composition, but also on the front mentioned. $\mathrm{A}$ is the inference function of cloud manufacturing service system, not only can save a lot of time to optimise the allocation of resources, it also can more accurate understanding the users' need in the original basis, to avoid artificial or service composition process error.

In the service have a back to pick up the premise of service B, behind of service B is the sequentially single service $\mathrm{X}$ or concurrent access service $\mathrm{X}$ and $\mathrm{Y}$, which can be according to the requirements and the given data base to carry out reasoning. Due to the combinable relationship of reasoning, may be it will involve the all combinable relations, which require huge combinable relations theory as a basis, but also need the actual situation analysis ability and sufficient intelligence automation. At present, there is a little combination of reasoning in domestic, on the one hand, because of the variety and reasoning results are not necessarily reliability, it is difficult to carry out a unified description; on the other hand, because it need services intelligent automation support, and the middle of a large number of complex relationship among the conversion, does not do too much exposition, as it shown in Figure 4.

Figure 4 The inference of the combinable relations

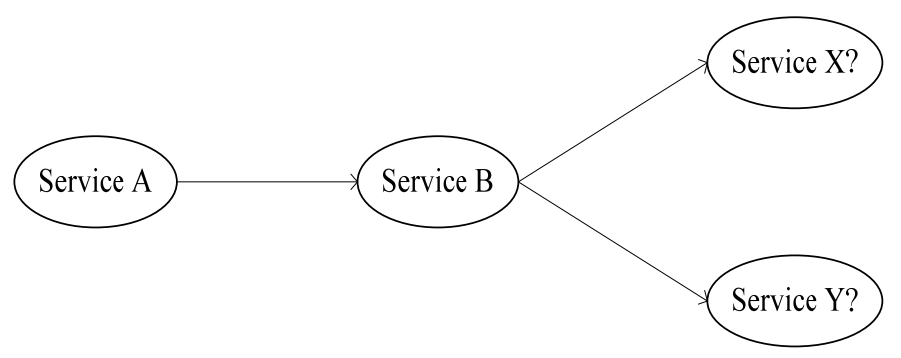




\section{Modelling of combinable relations based on protégé}

The ontology-based description language can be used to clearly describe the concept and various relations between concepts. The following specific description of the service ontology meta model are needed in a bearing production process in detail, its basic ontology meta model is shown in Figure 5.

Figure 5 Combined relationship ontology meta model sample (see online version for colours)

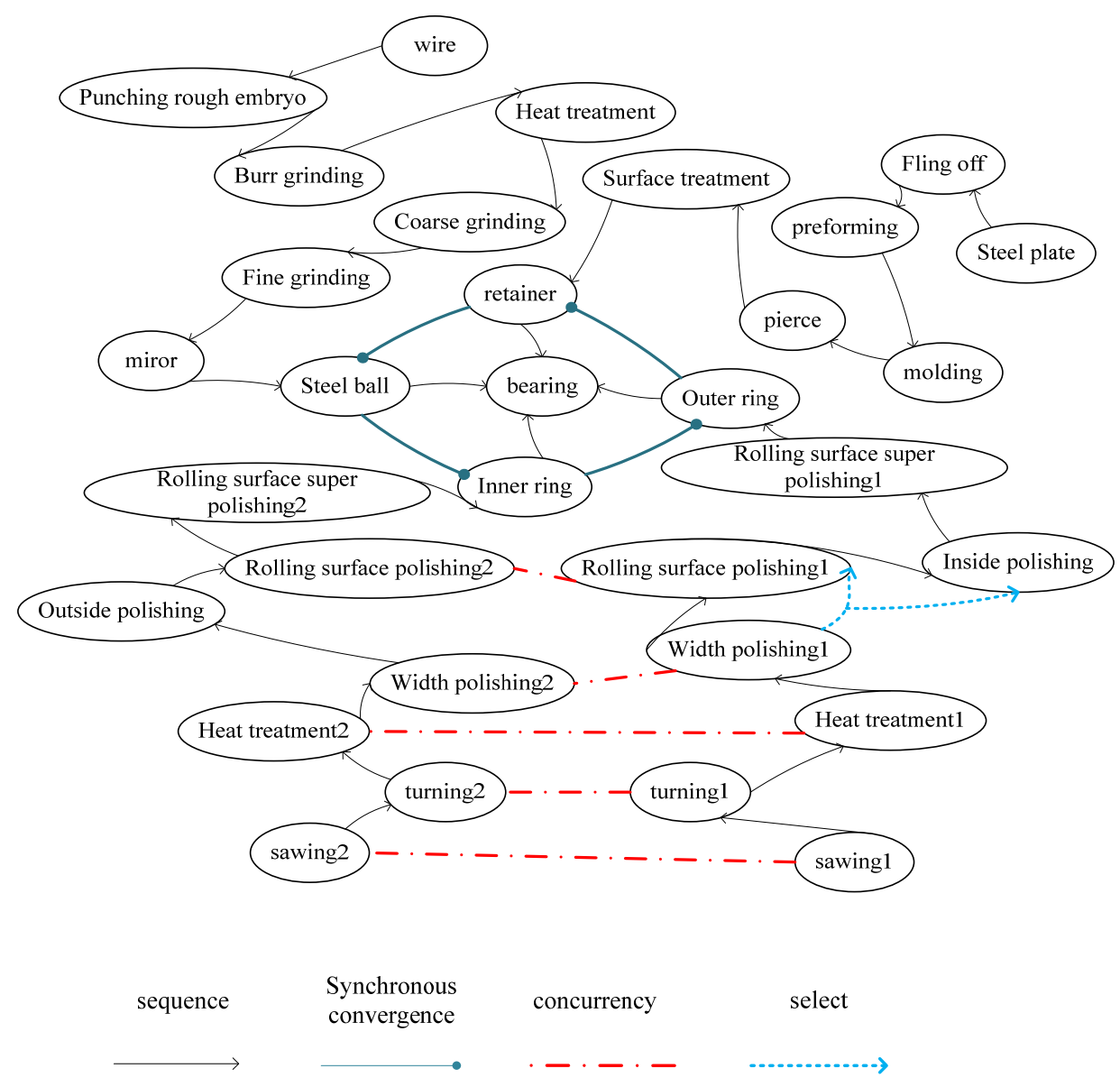

Step 1 Sawing off the cup and cone, and the heat treatment can be combined as a concurrent relationship.

Step 2 Outer ring, inner ring, retainer and steel ball need to be synchronised to form a bearing.

Step 3 Four parts of the bearing from the beginning of the raw material to the final product components are sequential relationships. 
Step 4 After finishing the width of the outer ring grinding, how to satisfied with the diameter of grinding conditions on the inside diameter grinding, how to meet the rolling surface grinding conditions on the rolling surface grinding.

\section{Conclusions}

Manufacturing services combination technology is a key technology of SOM theory. By combining various manufacturing resources or services, it will complete sophisticated manufacturing task and meet user needs. How to combine the manufacturing services, whether services can be combined, are premise conditions, the relationship among services is still relatively vague.

So this paper focus on the combinable relations, research on combinable relation classification by workflow and strength degree, such as sequential relationships branch/selection relationships, concurrent relationships, multiple selection, synchronous convergence, discriminator, arbitrary cycle, and delay selection. Finally, we uses OWL language to build a model of relations ontology for magnetic bearing.

However, the research is not comprehensive enough, because there are various kinds of combinable relations. At present, the ontology modelling technology is not perfect, especially for the cloud manufacturing service description. Although this paper superficial illustrates service relationship modelling, but at present, there has not been a unified standard way to describe relationships. So in the later work, we will make further research on the above aspects, and use the protégé tool to edit the ontology.

\section{Acknowledgements}

This work is sponsored by National Natural Science Foundation of China (No. 51505350). The Key Laboratory of Metallurgical Equipment and Control of Education Ministry, Wuhan University of Science and Technology (No. 2015B02). Sincere appreciation is extended to the reviewers of this paper for their helpful comments.

\section{References}

Ardagna, D. and Pernici, B. (2007) 'Adaptive service composition in flexible processes', Software Engineering, IEEE Transactions, Vol. 33, No. 6, pp.369-384.

Bohu, L. et al. (2010) 'An introduction to cloud manufacturing', ZTE Communications, Vol. 8, No. 4, pp.6-9.

Changjun, S. (2006) Research on Web Service Composition Relationship and its Implementation, Hohai University, Nanjing.

Changxia, W., Guanyu, L. and Buwei, C. (2009) 'Current situation and development trend of research on ontology construction tool', Computer \& Modernization, Vol. 1, No. 7, pp.26-28

Chunyang, Y., Xun, X. and Yuqian, L. (2015) 'Computer-integrated manufacturing, cyber-physical systems and cloud manufacturing-concepts and relationship', Manufacturing Letters, Vol. 6, pp.5-9. 
Feng, X. (2013) Research on Key Technology of Service Composition Based on Energy Consumption in Cloud Manufacturing System, Doctoral dissertation, Wuhan University of Technology.

Feng, X., Dinghua, Z., Ming, W. et al. (2014) Study on Semantic Description of Cloud Resources Based on Cloud Manufacturing Platform, Computer Engineering \& Applications.

Guo, H., Zhang, L., Tao, F. et al. (2010) 'Research on the measurement method of flexibility of resource service composition in cloud manufacturing', Advanced Materials Research, pp.139-141, 1451-1454.

He, J.F. (2008) 'Automatic web services composition based on reasoning Petri Net', International Conference on Advanced Language Processing and Web Information Technology, IEEE Computer Society, pp.569-574.

Kai, J.S., Hao, J., Wenting, X. et al. (2014) 'Consider the implementation of the reliability of cloud manufacturing service composition algorithm', Journal of Computer Aided Design and Graphics, Vol. 26, No. 3, pp.392-400.

Liu, B. (2012) Multi Task Oriented Service Composition and Optimization Technology in Cloud Manufacturing Environment, Chongqing University, Chongqing.

Nan, C. (2010) Research on Related Technologies of Semantic Web Service Composition, Central South University, Hunan.

Qing, Y. (2008) Research on Web Service Discovery Based on OWL-S, Nanjing University of Aeronautics and Astronautics, Nanjing.

Sheng, W.J., Jun, L.Z. and Jun, L.M. (2008) 'Composing semantic web services with description logics', Journal of Software.

Song, G., Chunhua, L. and Minglin, Z. (2011) 'Research on the implementation framework of semantic web service composition', Journal of Xinyang Normal University(Natural Science Edition), Vol. 24, No. 4, pp.536-540.

Tao, F. (2012) Research on Manufacturing Cloud Service Composition based on Extended HTN, Chongqing University, Chongqing.

Tao, F., Guo, H., Zhang, L. et al. (2012) 'Modelling of combinable relationship-based composition service network and the theoretical proof of its scale-free characteristics', Enterprise Information Systems, Vol. 6, No. 4, pp.373-404.

Tao, F., Lin, Z., Guo, H. et al. (2011) 'Research on key issues of cloud manufacturing characteristics and cloud service composition', Computer Integrated Manufacturing Systems, Vol. 17, No. 3, pp.477-486.

Tao, F., Zhao, D., Hu, Y. et al. (2008) 'Resource service composition and its optimal-selection based on particle swarm optimization in manufacturing grid system', IEEE Transactions on Industrial Informatics, Vol. 4, No. 4, pp.315-27.

Tong, Z. (2008) Research on The Key Technologies of Service Oriented Semantic Combination Simulation, National Defense Science and Technology University, Hunan.

Xudong, C. (2013) Classification and Management of Manufacturing Service Resources Based on Ontology in Cloud Manufacturing, Doctoral dissertation, Hubei University of Technology.

Xuejiao, W. and Xianhui, L. (2012) Cloud Manufacturing Service Description Based on Semantic, Vol. 1, No. 1, pp.40-43.

Yiming, L. (2013) Complex Multi Task Manufacturing Cloud Service Composition Based on Crossover and Mutation Particle Swarm Optimization, Doctoral dissertation, Chongqing University.

Zhang, L., Guo, H., Tao, F. et al. (2010) 'Flexible management of resource service composition in cloud manufacturing', in Industrial Engineering and Engineering Management (IEEM), 2010 IEEE International Conference, 7-10 December, pp.2278-2282.

Zhen, C., Dechen, Z. and Hanchuan, X. (2013) Ontology Based Resource Virtualization in Cloud Manufacturing Environment, Harbin Industrial University, Heilongjiang. 
Zhiwei, L., Ning, M. and Qingxin, C. (2010) Research on the Knowledge Ontology Description Method of Tire Mold Technology, Guangdong University of Technology, Guangdong.

Zhiyao, T., Gang, C. and Jinxiang, D. (2006) Network Manufacturing Ontology Construction Analysis Based on OWL, Zhejiang University, Zhejiang. 